\title{
Modelo de evaluación de mercado: Herramienta para decisiones de negocios
}

\author{
Recepción: Mayo de 2006 / Aceptación: Junio de 2006
}

(1) José Porlles Loarte

(2) Julio Yenque Dedios

(3) Aurelio Lavado Soto

\section{RESUMEN}

En el presente trabajo se analizan los conceptos de mercado potencial y mercado real como base para decisiones estratégicas de mercado con visión de largo plazo cuando se evalúa la implantación de un negocio en cierta área geográfica. Sobre este marco conceptual, se propone la herramienta metodológica para evaluar una decisión comercial, para lo cual se toma como referencia el caso de la industria cervecera en el Perú, considerando que esta industria enfrenta en la región reordenamientos empresariales dentro de una estrategia de crecimiento.

Palabras Clave: Mercado potencial, mercado global, consumo per cápita.

\section{MARKET EVALUATION MODEL: TOOL FOR} BUSINESS DECISIONS

\section{ABSTRACT}

In the present work the concepts of potential market and global market are analyzed as the basis for strategic decisions of market with long term perspectives, when the implantation of a business in certain geographic area is evaluated. On this conceptual frame, the methodological tool is proposed to evaluate a commercial decision, for which it is taken as reference the case from the brewing industry in Peru, considering that this industry faces in the region entrepreneurial reorderings within a growth strategy.

Key words: Potential market, global market, per capita consumption.

\section{INTRODUCCIÓN}

En el desarrollo de estudios de inversión, el análisis de mercado es vital para asegurar la solidez y viabilidad comercial de una opción de inversión. De sus resultados depende toda la construcción posterior del plan de negocios. Una falla en la apreciación del desarrollo futuro del mercado puede ser catastrófico para el inversionista, de modo que se requieren herramientas de análisis que aseguren una buena fundamentación para la decisión comercial. Obviamente que el empresario o analista utilizará las herramientas disponibles de acuerdo a la naturaleza y envergadura del proyecto bajo análisis.

Como es natural en la formulación del estudio de mercado se tiene que abarcar todos los puntos al detalle como: la definición del producto, precios, segmentos de mercado, canales de distribución, formas y mecanismos de promoción y publicidad, entre otros aspectos. Sin embargo, previo al desarrollo frontal de estos tópicos, se pueden utilizar criterios previos para apreciar la potencialidad y dinámica del mercado objeto de interés. Y esto es lo que hacen o requieren los empresarios. Apreciar las perspectivas de crecimiento del consumo per cápita, parámetro que está vinculado al desarrollo del mercado potencial, vale decir, preguntarse ¿Hay mercado potencial, cuán expectante es el negocio?.

Este es el propósito del presente trabajo, integrar diferentes conceptos que ya existen en un modelo consolidado y coherente que sirva como herramienta metodológica para la evaluación de decisiones comerciales con perspectivas de largo plazo.

\section{MERCADO POTENCIAL Y MERCADO REAL (EFECTIVO)}

El uso de los conceptos de mercado potencial, mercado real (mercado efectivo o global) y mercado objetivo, así como su adecuada interpretación permitirá proporcionar la solidez necesaria para la toma de decisiones en aspectos de mercado.

\section{¿Cómo se interpreta el mercado potencial?}

Es el nivel máximo de demanda de un bien, en la hipotética situación en que todos los consumidores posibles se constituyeran en consumidores reales. En consecuencia, la demanda potencial de un bien es demanda que existe en un ámbito territorial para el consumo de dicho producto, pero que la presencia de diversas fuerzas contrarias, impiden cubrir las

\footnotetext{
(1) Magíster en Administración. Profesor del Departamento de Análisis y Diseño de Procesos, UNMSM. E-mail: joseporlles@yahoo.com

(2) Ingeniero Industrial. Profesor del Departamento de Diseño y Tecnología Industrial, UNMSM. E-mail: jyenque@yahoo.es

(3) Ingeniero Químico. Profesor del Departamento de Diseño y Tecnología Industrial, UNMSM. E-mail:mlavados@unmsm.edu.pe
} 


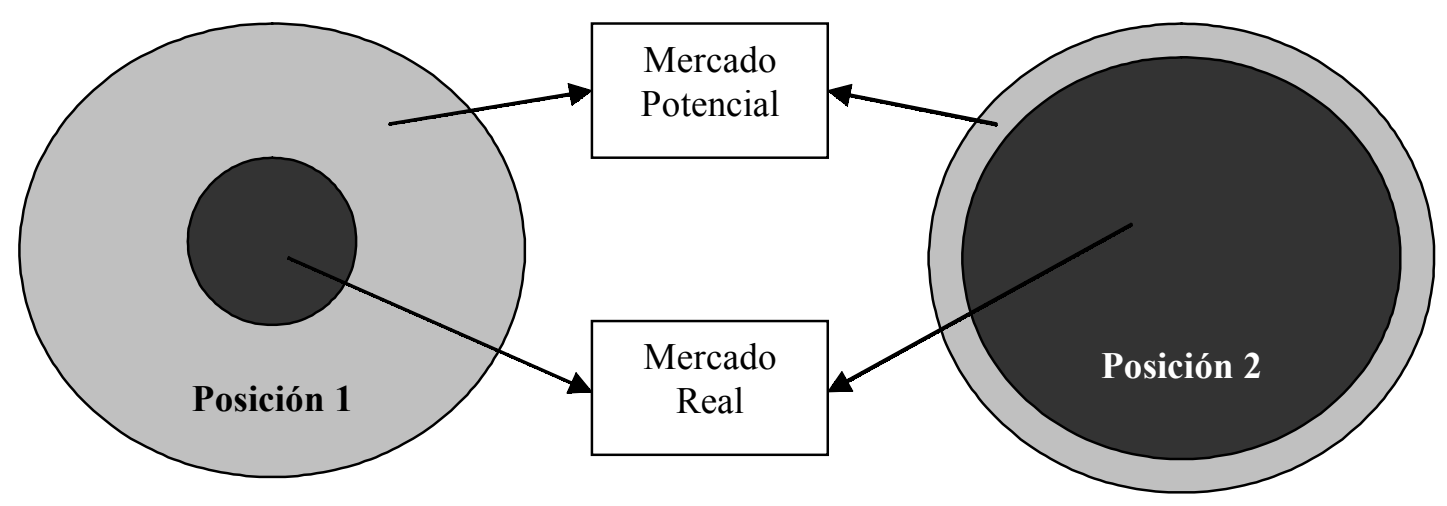

Figura 1. llustración del mercado potencial y mercado real

necesidades del consumidor. Este concepto proporciona una magnitud de la demanda futura de un producto en un país o región, teniendo en cuenta la posición relativa en relación con países de similar nivel de desarrollo. Esta situación se puede medir con la demanda per cápita en cada país o región respectivamente. En términos relativos una economía puede presentar dos posiciones como se visualiza en la Figura 1.

La comparación relativa grafica que en la posición 1 el país configura una demanda potencial importante en relación a la demanda real, lo que se traduce como que el consumo per cápita es bajo en relación a un referente similar. Lo anterior significa que la demanda real del mercado de un producto no se ha expandido en términos relativos en proporción a su población. La explicación es que en la práctica no es cubierta debido a la presencia de múltiples factores, entre los cuales se pueden citar: precio relativamente alto para la capacidad de compra del promedio de la población, sustitutos más asequibles, poca competencia de los ofertantes por el lado del precio, ausencia de medios de marketing contundentes para incrementar la compra o bien sus efectos están bloqueados por la baja capacidad adquisitiva del mercado.

Pero si de alguna manera se modifica la presión de algunos de dichos factores, se cambia favorablemente la dinámica de las fuerzas imperantes promoviendo el crecimiento del consumo. En este escenario, el riesgo de incursionar en el negocio es potable y manejable, tanto en lo que se refiere para justificar una ampliación de planta para los productores ya establecidos o el ingreso de nuevos industriales. Además es posible que puedan ingresar más de un inversionista. Las barreras de mercado que impongan los industriales ya establecidos, pueden ser superables y los negocios prometedores.
El caso es diferente en la posición 2. La brecha entre el mercado real y el mercado potencial no estimula nuevas inversiones en este mercado. El ingreso a estos mercados es altamente riesgoso, si no se cuenta con una sólida capacidad económica para sostener posibles "guerras" con la competencia en funcionamiento. Es muy improbable o es muy costoso lograr que la aplicación de determinadas estrategias de marketing pueda promover un mayor consumo, salvo que la capacidad adquisitiva de la población mejore sustantivamente, lo que es remoto en países como es el caso del Perú.

\section{LA INDUSTRIA DE CERVEZA EN EL PERÚ}

En la actualidad la U.C.P. Backus \& Johnston registra más de 100 años en el mercado peruano y durante ese periodo la familia Bentín de nacionalidad peruana detentó el control accionario de la compañía. Sin embargo, derivado del proceso de consolidación regional que viene enfrentando la industria cervecera, durante el segundo semestre 2002 el Grupo colombiano Bavaria toma el control de la compañía, debiendo destacarse que dicha empresa es la segunda más grande a nivel latinoamericano. Se estima que el mercado cervecero representa 400 millones de dólares en venta.

El Grupo Backus hasta el 2004 continuaba como líder de la producción de cervezas en el Perú dado que controla todas las plantas distribuidas en el país con las marcas: Cristal, Pilsen, Cusqueña, Arequipeña y San Juan, con una capacidad instalada total de 10,3 millones de hectolitros, de la cual la planta de Ate representa 5,2 millones de hectolitros (HI) de capacidad (12). A diciembre 2003 el uso de la capacidad del Grupo ascendió a $61 \%$ y de la planta de Ate a 71\%. A diciembre 2004 el Grupo hizo uso del $66 \%$ de su capacidad instalada total y el $73 \%$ de 
Cuadro 1. Perfil de las marcas de cervezas en el mercado peruano

\begin{tabular}{|l|c|c|c|c|c|c|c}
\hline Marca & Cristal & $\begin{array}{c}\text { Pisen } \\
\text { Callao }\end{array}$ & Cusqueña & Arequipeña & $\begin{array}{c}\text { Pisen } \\
\text { Trujillo }\end{array}$ & $\begin{array}{c}\text { San } \\
\text { Juan }\end{array}$ & Otras \\
\hline $\begin{array}{l}\text { Participación } \\
(\%)\end{array}$ & $56,1 \%$ & $20,2 \%$ & $9,3 \%$ & $5,0 \%$ & $4,9 \%$ & $1,8 \%$ & $2,3 \%$ \\
\hline
\end{tabular}

Fuente: Unión de Cervecería Peruanas Backus \& Johnston-UCPBJ, 2004.

su planta de Ate. En el Cuadro 1 se presenta el perfil de participación de mercado de las marcas más importantes de cerveza a diciembre 2003.

El Grupo Backus resalta que ha convertido la Planta de Ate en una de las plantas cerveceras más modernas en América Latina, habiendo invertido desde su construcción en 1976 hasta 2002 cerca de 219 millones de dólares americanos, pasando de una capacidad instalada de 3,9 a 5,2 millones de $\mathrm{HI}$.

A lo largo del periodo 1996-2001 la producción de cerveza blanca del Grupo Backus (que es la línea principal en relación a la cerveza negra) se redujo en $28 \%$, aumentando en el 2002 en $17 \%$, logrando alcanzar una producción de 606 millones de litros (It); similar comportamiento se refleja en la producción de cerveza negra. La evolución de la producción de la cerveza blanca se aprecia en la Figura 2.

El consumo de cerveza está influenciado de manera similar que la gaseosa por dos factores: el poder adquisitivo de la población y por el precio del producto; factores que han influenciado en la tendencia negativa de la producción, que se ha revertido en el 2003. En el Perú, el factor precio ha sido altamente influenciado por los impuestos en especial del Impuesto Selectivo al Consumo (ISC), que fue modificado de variable a fijo en 1997, ajustable periódicamente el cual fue en aumento llegando a 1,31 nuevos soles por litro en septiembre 2002 (0,38 US\$/It), constituyéndose uno de los impuestos más altos de la región.

El consumo per cápita de cerveza en el Perú, América Latina y el Mundo ¿Cómo era el consumo per cápita hasta el periodo 2003-2004?. En términos relativos el nivel per cápita de consumo de cerveza era y es baja en el Perú como en América Latina, en comparación con los otros países de la región y lejos de los niveles que se registran en Europa. En promedio en el 2001, América Latina $(A L)$ registró 30 It/hab/año, comparado con España (66 lt), Alemania (130 lt), en tanto que en el Perú el promedio representaba 22 lt/hab/año, indicador relevante que mostró estabilidad en los últimos años 2001-2003 (Figuras 2 y 3).

De acuerdo a la Figura 5, el consumo per cápita del Perú era de 30,0 litros (It) en 1993, 31,8 It en 1996, disminuyendo sostenidamente a 19,7 It hasta el 2001 , lo que demuestra la caída en las ventas tota-

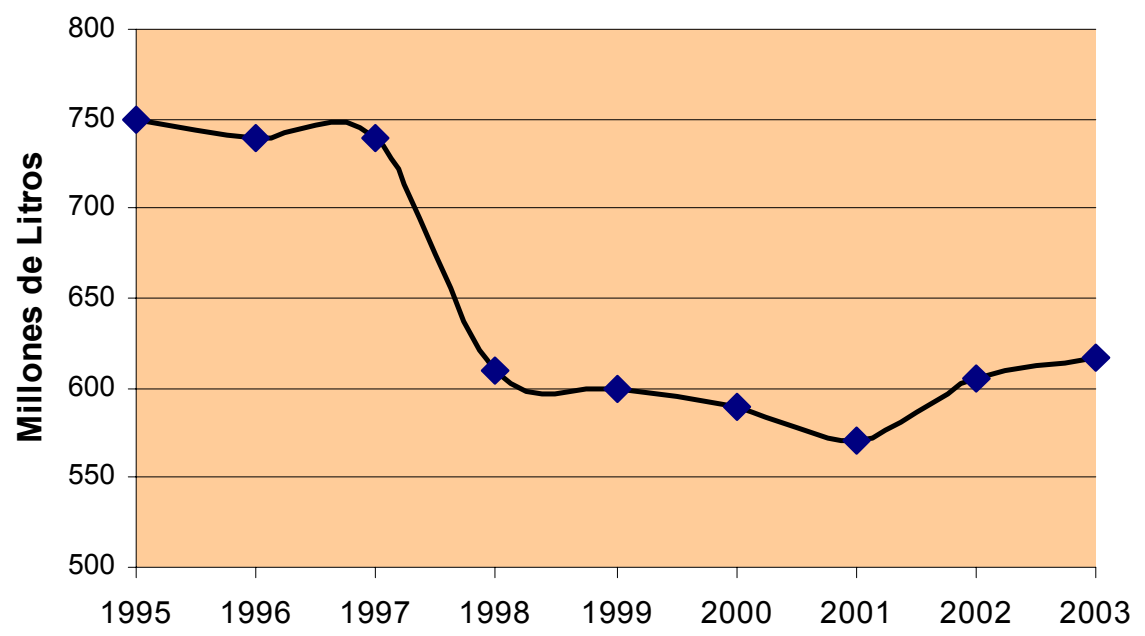

Figura 2. Producción de cerveza en el Perú Fuente: Maximixe. El Comercio del 27.04.03 


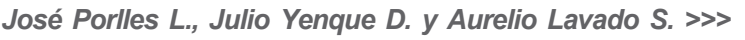

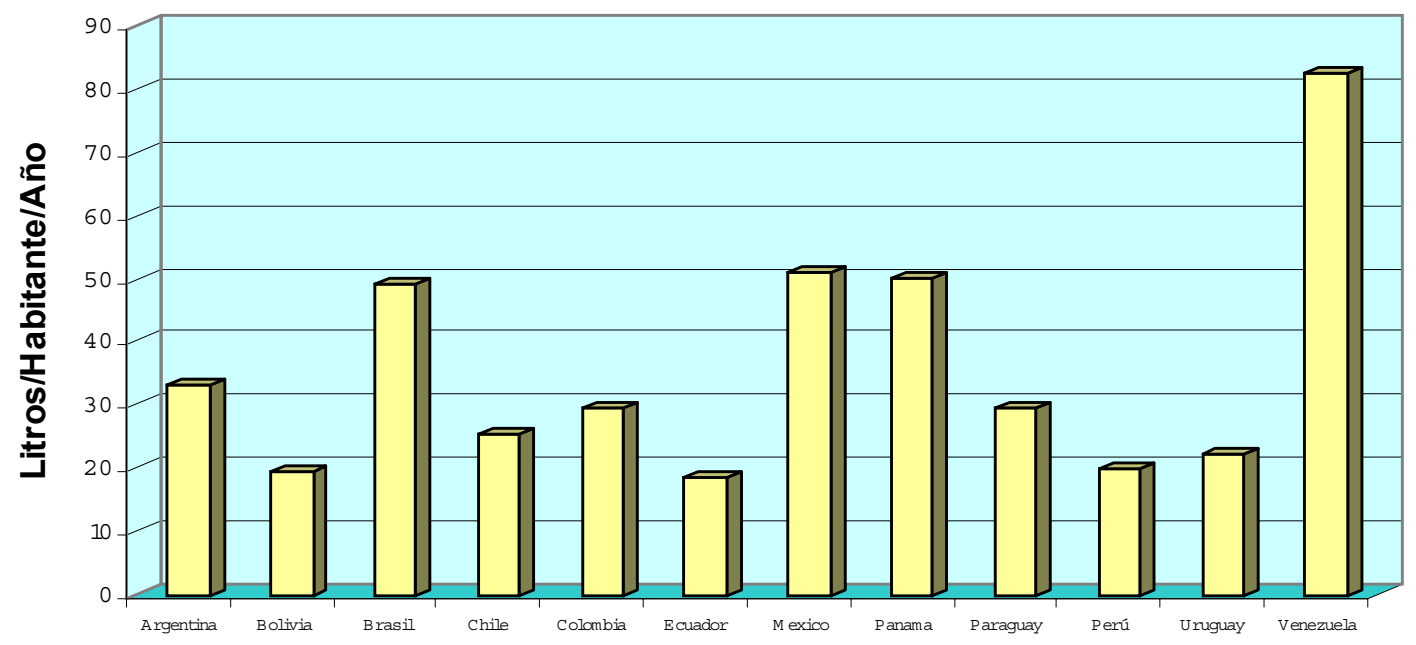

Figura 3. Consumo de cerveza per cápita en Latinoamérica

Fuente: Wong, R. Grupo Cervecero B\&J-Innovación Estratégica, 2004

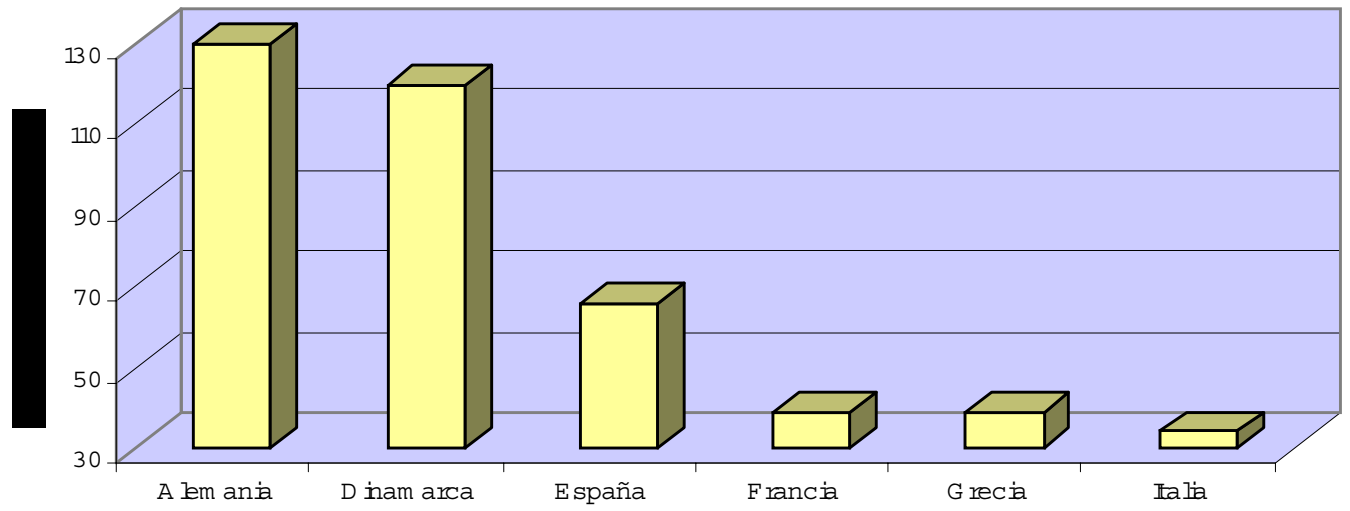

Figura 4. Consumo de cerveza per cápita en Europa

Fuente: Asociación Latinoamericana Fabricantes de Cerveza, 2001

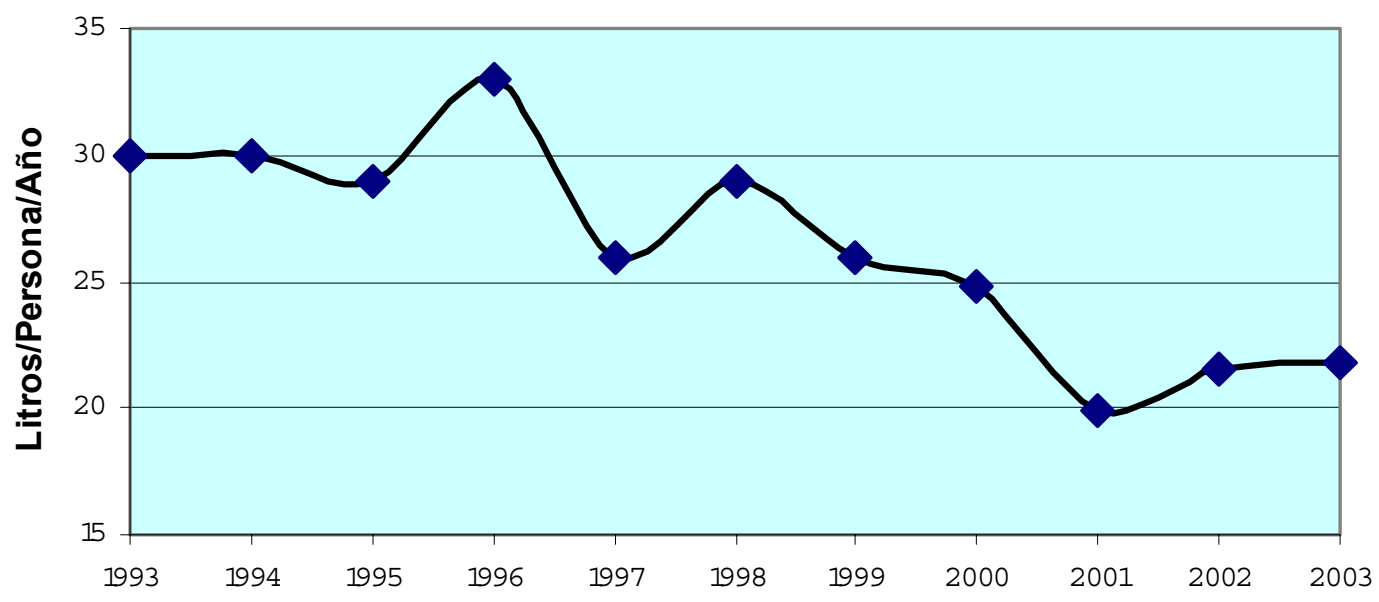

Figura 5. Evolución consumo per cápita de cerveza en el Perú Fuente: Maximixe. El Comercio del 27.04.03 
Cuadro 2. Principales cervecerías del mundo (2003)

\begin{tabular}{|r|l|l|r|r|}
\hline \multicolumn{5}{|c|}{ Las 15 mayores cervecerías del mundo (2003) } \\
\hline \multicolumn{1}{|c|}{ Cervecería } & País & $\begin{array}{c}\text { Producción (Mill } \\
\text { HI) }\end{array}$ & $\begin{array}{c}\% \text { de la Prod. } \\
\text { Mundial }\end{array}$ \\
\hline 1 & Anheuser-Bush & USA & 152,0 & $10,3 \%$ \\
\hline 2 & SAB-Miller & Sudáfrica-USA & 137,8 & $9,3 \%$ \\
\hline 3 & Heineken & Holanda & 99,0 & $6,7 \%$ \\
\hline 4 & Interbrew & Bélgica & 97,9 & $6,6 \%$ \\
\hline 5 & Carlsberg & Dinamarca & 88,8 & $6,0 \%$ \\
\hline 6 & Ambev & Brasil & 67,4 & $4,6 \%$ \\
\hline 7 & Modelo & México & 41,9 & $2,8 \%$ \\
\hline 8 & Coors & USA & 38,6 & $2,6 \%$ \\
\hline 9 & Tsingtao & China & 32,6 & $2,2 \%$ \\
\hline 10 & Scottish \& Newcastle & Reino Unido & 31,8 & $2,2 \%$ \\
\hline 11 & Asahi & Japón & 25,9 & $1,8 \%$ \\
\hline 12 & Femsa (Cuauhtemoc) & México & 24,6 & $1,7 \%$ \\
\hline 13 & Santo Domingo (Bavaria) Colombia & 23,5 & $1,6 \%$ \\
\hline 14 & Kirin & Japón & 23,1 & $1,6 \%$ \\
\hline 15 & Yan Ying & China & 22,3 & $1,5 \%$ \\
\hline Fuente: ALAFACE. Indice 2005 Información Estadística de la Industria \\
Cervecera. (Www.cerveceroslatinoamericanos.com) \\
\hline
\end{tabular}

les producto del ciclo recesivo que experimentó la economía peruana. En el 2003 se evidencia una recuperación a 22,4 It., nivel que se ha mantenido durante el 2004.

\section{Elasticidad precio de la demanda}

Es reconocido por los especialistas que la razón para que los peruanos no beban tanta cerveza es básicamente por el precio de la bebida. En efecto, está demostrada la fuerte relación entre menor precio y el aumento del consumo, como es el caso de la tradicional cerveza de $620 \mathrm{ml}$. La empresa Maximixe (6) confirma que la demanda de esta bebida en el Perú es elástica; lo que significa que una disminución del $1 \%$ en el precio refleja un incremento importante del consumo. La misma fuente anota que, “...mediante un estudio se demostró que si se redujera en $18 \%$ los precios actuales de la cerveza, el mercado crecería en $30 \%$ ". Entonces, según dicho estudio significa que la elasticidad tiene un valor de 1,67.

En el caso peruano durante el 2004, cuando se bebe una botella de cerveza de $620 \mathrm{ml}$, se está pagando $27,8 \%$ de ISC, vale decir 1,45 nuevos soles por cada botella en tienda de 3,50 nuevos soles antes del IGV. Según ejecutivos de Backus (7) lo ideal es que dicho impuesto se reduzca a la tercera parte, lo cual aumentaría la demanda significativamente. Re- fieren “... en el año 2000 se redujo el ISC y las ventas crecieron en más de $12 \%$ ".

Otro factor que hace que el mercado se configure como muy apetitoso es el estudio realizado por el Grupo Apoyo (8). Según sus cifras el $50 \%$ de las bebidas alcohólicas consumidas en el Perú son de origen informal, mientras que el otro $50 \%$ del mercado es cubierto por la cerveza (44\%) y los licores formales (6\%). Asimismo, un reciente estudio de la empresa de investigación CCR (9) indica “... los peruanos entre los 18 y 35 años de edad prefieren la cerveza, y que este producto es el que más se vende en las bodegas". Ejecutivos de CCR opinan que definitivamente el ingreso de una nueva marca al mercado hará crecer las cifras de consumo por la novedad y la influencia de la publicidad.

La competencia en el mercado peruano ¿Qué motivó a la empresa Cervecera Brasileña Ambev incursionar en el Perú, considerada la sexta mayor cervecera del mundo con una participación mundial que bordea el $5 \%$ en el 2003, como se aprecia en el Cuadro 2?. El principal atractivo que vieron los brasileños en el mercado peruano fue que el consumo per-cápita de cerveza al año es de solo 22 litros, cuando hace menos de diez años se había registrado cerca de 35 litros, y en 1987 se creció a 42.

La gerencia de AmBev (10) señalaba que el Perú tiene la cerveza más cara de Sudamérica, añadiendo que esta situación se debe a la existencia de un solo jugador en la cancha, además de los altos impuestos. Resalta además que la competencia va a generar que el mercado fije precios más bajos como sucedió en el caso de los celulares, aunque advierte que no puede decir a cuánto disminuirá. El Perú es el país sudamericano donde la cerveza (botella de $620 \mathrm{ml}$ ) tiene el mayor precio sin consignar la carga tributaria tal como se muestra en el Cuadro 3.

\section{MERCADO REAL O EFECTIVO}

Este concepto se refiere a la demanda que se registra en términos estadísticos de acuerdo a las condiciones del entorno que rigen en el mercado. Es la

Cuadro 3 . Precios de botella de cerveza en Sudamérica (sin incluir impuestos)

\begin{tabular}{|c|c|c|c|c|c|c|c|c|c|c|}
\hline País & Perú & Colombia & Bolivia & Venezuela & Uruguay & Ecuador & Chile & Brasil & Paraguay & Argentina \\
\hline $\begin{array}{c}\text { Precio } \\
\text { US\$ }\end{array}$ & 0,415 & 0,335 & 0,326 & 0,319 & 0,318 & 0,317 & 0,316 & 0,257 & 0,252 & 0,139 \\
\hline
\end{tabular}

Fuente: Diario El Comercio del 03.06.04 y 02.12 .04 
Cuadro 4. Ventas previstas de la Industria Cervecera

\begin{tabular}{|l|c|c|c|c|c|c|}
\hline Concepto & Año base & Año 1 & Año 2 & Año 3 & Año 4 & Año 5 \\
\hline Año & 2003 & 2004 & 2005 & 2006 & 2007 & 2008 \\
\hline $\begin{array}{l}\text { Demanda } \\
\text { (millones de It) }\end{array}$ & 602,7 & 614,8 & 627,1 & 639,6 & 652,4 & 665,5 \\
\hline
\end{tabular}

Fuente: Elaboración propia, considerando 2\% de crecimiento anual. 2006

cantidad del bien que realmente está siendo colocado o adquirido en el mercado. Desde otro enfoque, es la demanda constituida por ciertos consumidores que tienen las condiciones tangibles necesarias para consumir cierto producto.

El mercado peruano registró en el 2003 una demanda de 602,7 millones de It (11) para una población de 27,15 millones de habitantes. Los expertos pronosticaban (4) en ese entonces que el mercado cervecero de Lima crece a tasas del $2 \%$ anual en las condiciones del entorno imperantes en dicho periodo. En términos conservadores se asume que se mantendrá esta tendencia, entendido como los niveles más probables de ventas durante un periodo determinado. Estas perspectivas son muy independientes del mercado potencial analizado.

Entonces, manteniendo las referidas condiciones de mercado, en el Cuadro 4 se presenta una proyección del mercado real o efectivo entendido como ventas previstas de la actividad para los próximos cinco años con una previsión del $2 \%$ de crecimiento promedio anual.
RELACIÓN DEL MERCADO REAL Y POTENCIAL

Si en el análisis previo se identifica una brecha interesante entre ambos mercados, entonces de debe evaluar el futuro con un sentido de prospectiva, vale decir, que uno construye el escenario de mercado deseado o lo transforma en función de las capacidades y fortalezas para lograrlo. Es claro que las metas propuestas conlleva un plan de acción que incluye medidas para dinamizar el mercado real, tales como la introducción de un menor precio que los competidores, mecanismos de márketing agresivos para impulsar una mayor frecuencia de compra de los consumidores, entre otras acciones.

Para apreciar cuán cerca o lejos se encuentran las proyecciones del mercado real en relación al mercado potencial, se procede a analizar las perspectivas de incremento del consumo per-cápita como meta, para lo cual se establecen tres escenarios posibles de lograr en los próximos cinco años (Cuadro 5), según los criterios siguientes:

- Escenario 1: Alcanzar un consumo per cápita mínimo de 25 It/hab/año (criterio muy conservador).

Cuadro 5. Análisis de escenarios futuros

\begin{tabular}{|l|c|c|c|}
\hline Conceptos & Escenario 1 & Escenario 2 & Escenario 3 \\
\hline Meta al año 5 & 25 It per-cápita & 30 It per-cápita & 35 It per-cápita \\
\hline $\begin{array}{l}\text { Población actual } \\
\text { (millones de Habit.) }\end{array}$ & $27 ; 15$ & 27,15 & 27,15 \\
\hline $\begin{array}{l}\text { Tasa de crecimiento } \\
\text { Poblacional }\end{array}$ & 1,6 & 1,6 & 1,6 \\
\hline Población al año 5 & 29,4 & 29,4 & 29,4 \\
\hline $\begin{array}{l}\text { Mercado potencial al año 5 } \\
\text { (millones It) }\end{array}$ & $\mathbf{7 3 5}$ & $\mathbf{8 8 2}$ & $\mathbf{1 , 0 2 9}$ \\
\hline Mercado Global año 5 & $\mathbf{6 6 6}$ & $\mathbf{6 6 6}$ & $\mathbf{6 6 6}$ \\
\hline $\begin{array}{l}\text { Diferencias en el año 5 } \\
\text { (mill It) }\end{array}$ & $\mathbf{6 9}$ & $\mathbf{2 1 6}$ & $\mathbf{3 6 3}$ \\
\hline
\end{tabular}

Anotación importante: Se han mantenido los tres escenarios señalados por Porlles, J., en el documento "Modelo de Evaluación de Mercado: Una Alternativa para Decisiones de Negocios", un trabajo inicialmente formulado y publicado en Internet por Monografías.com en Diciembre 2004. 
- Escenario 2: Alcanzar un consumo per cápita de

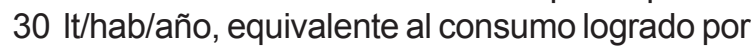
el Perú en 1993 y similar al promedio latinoamericano ya anotado.

- Escenario 3: Alcanzar un consumo per cápita de 35 It/hab/año, equivalente al consumo de Argentina en el 2004 (2) y mucho menor del 42 logrado por el Perú en 1987.

Como se aprecia, la meta de consumo per-cápita de 25 It que representa $12.6 \%$ de incremento al consumo per-cápita de 22.2 It, sería aproximadamente alcanzado por el crecimiento del mercado real actual si persisten las condiciones actuales de un solo productor que controla el mercado en una posición monopólica, sin disminución importante del precio por parte del productor.

Para una meta de 30 It representa un incremento del $35 \%$ del consumo per-cápita de 22,4 It que todavía está lejos de los países vecinos y del promedio de la región, no sería logrado con las previsiones del mercado real actual, lo que significa que se vislumbra un techo importante a alcanzar y que los negocios que hoy se realicen tienen un riesgo mínimo de fracaso.

De imponerse una modificación estructural del mercado peruano con el ingreso de nuevos productores, con un menor precio y con una agresiva promoción del producto es posible alcanzar la meta de 35 It percápita que representa un $58 \%$ de incremento del indicador 22,2 It. Si este cambio se da con la ampliación de nuevas formas estratégicas de expansión de la demanda total de consumo de cerveza, entonces el mercado real podría crecer a un ritmo mucho más alto que el $2 \%$ previsto en dichas circunstancias, y dado el techo que se tiene con el referido mercado potencial se da por descontado prácticamente el éxito de los negocios que incursionen en esta actividad.

\section{EL MERCADO OBJETIVO}

Este mercado es la porción o cuota del mercado real que le correspondería a una empresa ya instalada, o de una nueva que pretende alcanzar la demanda futura proyectada, entendido como el pronóstico de ventas de la citada empresa.

En el caso bajo análisis, podría significar como meta captar el $10 \%$ al $20 \%$ de la demanda global proyectada para el año 5 (usando un criterio conservador, puesto que el otro criterio sería usar el mercado potencial como referencia), lo que significaría pretender un mercado objetivo entre 66 a 120 millones de It. Considerando la importante diferencia existente en- tre los mercados real y potencial no se vislumbra riesgo que impida ingresar al negocio; muy por el contrario es factible lograrlo.

El grupo empresarial AmBev ha anunciado su decisión de instalar una planta de cerveza en HuachipaLima con una capacidad de 100 millones de lt al año ( 1 millón de HI/año) con una inversión estimada en 80 millones de dólares, con su producto marca Brahma.

\section{¿QUÉ HA SUCEDIDO A MARZO 2006?}

En una entrevista periodística en Noviembre 2004 (5) el Gerente de Márketing de Amveb Perú mencionaba “... que desde Octubre 2004 Amveb Perú es la nueva distribuidora de Másventas a través de las gaseosas del grupo Pepsico, y que a partir del 2005, Amveb empezará a distribuir además, su primera cerveza producida en el Perú, indicando además que en unos cinco o seis meses tardarán en construir la planta cervecera, es decir, si no nos ponen trabas".

En realidad, Amveb Perú inauguró oficialmente su planta con fecha 31 de Octubre del 2005, después de superar ciertas trabas que no es objeto de este trabajo comentarlas; debiendo resaltarse que la construcción demoró el tiempo récord de cinco meses en un terreno de 14,5 hectáreas.

En Perú, el mercado se ha visto animado desde el 2005, tras la entrada de Ambev. El mercado cervecero viene presentando un mayor dinamismo, registrando en el 2005 un aumento en ventas del 14\%, lo que configura que el consumo per cápita ha pasado de 20 It de cerveza en el 2001 a 28 It por año, cifra que se encuentra lejos del promedio latinoamericano último que supera a los 35 litros anuales (3).

En cuanto a la presencia de las dos empresas rivales en el mercado nacional (Backus y Ambev), Amveb Perú anuncia “... que ya cuenta con el $18 \%$ de participación de mercado en Lima, $20 \%$ en Trujillo y $15 \%$ en Huancayo; agregando que el precio de la cerveza registró una reducción de $15 \%$ en el 2005 , y para este año (2006) se podría observar una reducción similar" (1).

\section{CONCLUSIONES}

Del análisis efectuado se desprende que en el Perú se registra un gran mercado potencial de consumo de cerveza, teniendo en cuenta su relación con otros países de la región. Esta demanda potencial definitivamente encierra un volumen muy importante de de- 
manda latente, vale decir, que se evidencia una brecha importante entre la oferta instalada y la demanda potencial. Esto significa que cualesquier inversión adicional que se realice para incursionar en esta industria para incrementar la capacidad instalada actual, es un negocio potencialmente bueno en el largo plazo.

La presencia de Amveb en el Perú así lo confirma, abonando la tesis aquí planteada, cuando los ejecutivos de dicha empresa afirmaban que el consumo anual de cerveza per cápita de 22 It, era un nivel relativamente bajo en la región, por lo que era posible maximizar oportunidades futuras, y es lo que están haciendo.

La guerra de la cerveza ya se dio inicio y está en marcha. Se vislumbra un nuevo escenario de competencia en la industria de cerveza peruana, el planteamiento de nuevas estrategias (13) para hacer frente al nuevo entorno, que involucra un nuevo posicionamiento de Backus y una "guerra de precios" ya se refleja en la industria. El nuevo foco estratégico de competencia tendrá como efecto positivo el incremento de la demanda per cápita de cerveza en el mercado peruano, los indicadores últimos así lo demuestran.

\section{REFERENCIAS BIBLIOGRÁFICAS}

1. Acuerdo con empresa española (2006). Nota periodística publicada el 30.03.06 en el Diario El Comercio.

2. ALAFACE (2005). Índice 2005.Información Esta- dística de la Industria Cervecera (www.cerveceroslatinoamericanos.com).

3. Grupo Backus logra record de ventas en el mes de Diciembre (2006). Nota periodística publicada el 03.01.06 en el Diario El Comercio.

4. La Guerra de las Cervezas (2004). Economía \& Negocios, p. b1. Diario El Comercio del 10.10.04

5. Las Novedades de Ambev (2004). Revista Másventas, Año 4, Nro 37, Noviembre 2004, p. 24. Diario El Comercio.

6. Loayza, J. y López, C. (2004). Pulseo Chelero. Revista Dominical, pp. 10-11. Diario de La República del 28.03.04.

7. Loayza, J. y López, C., op. cit., p. 10

8. Loayza, J. y López, C., op. cit., p. 9

9. Loayza, J. y López, C., op. cit., p. 9

10.Loayza, J. y López, C., op. cit., p. 8

11. Maximixe (2003). Estadística de la Industria Cervecera, p. b7. Diario El Comercio del 27.04.03.

12. Maldonado, O. \& Izaguirre, J. (2004). Estudio Unión de Cervecería Peruanas Backus \& Johnston S.A.A. Apoyo \&Asociados, Mayo 2004.

13.Wong, R. (2004). Grupo Cervecero Backus \& Johnston- Innovación Estratégica. GestioPolis.com 